\title{
ANSIEDAD ESCOLAR INFANTOJUVENIL: UNA REVISIÓN DE AUTOINFORMES
}

\author{
Aitana Fernández-Sogorb \\ Universidad de Alicante - afs44@alu.ua.es \\ Ma Isabel Gómez-Núñez \\ Universidad Miguel Hernández de Elche \\ María Vicent \\ Universidad de Alicante \\ M. P. Aparicio-Flores \\ Universidad de Alicante \\ David Aparisi-Sierra \\ Universidad de Alicante \\ Cándido J. Inglés Saura \\ Universidad Católica San Antonio de Murcia \\ https://doi.org/10.17060/ijodaep.2017.n1.v3.973
}

Fecha de Recepción: 23 Enero 2017

Fecha de Admisión: 1 Abril 2017

\section{RESUMEN}

La ansiedad escolar es el conjunto de reacciones cognitivas, motoras y psicofisiolóficas emitidas por una persona ante situaciones del ámbito escolar que son percibidas como una amenaza. Los objetivos de investigación de este trabajo fueron dos. El primero de ellos fue describir los autoinformes de ansiedad escolar que han sido elaborados y/0 validados entre el año 2005 y el 2015: Inventario de Ansiedad Escolar, School Anxiety Inventory-Short Version, Children's Anxiety in Math Scale y Test Anxiety Inventory for Children and Adolescents. El segundo propósito consistió en analizar la fiabilidad (consistencia interna y estabilidad temporal) y validez (estructura factorial) de los inventarios y de la escala mencionados antes. Los resultados sugieren que las propiedades psicométricas de estos instrumentos son adecuadas. Por tanto, pueden ser usados por profesionales clínicos y educativos para evaluar la ansiedad escolar de niños y adolescentes.

Palabras clave: ansiedad escolar, infancia, adolescencia, evaluación, autoinforme.

\section{ABSTRACT}

Child and youth school anxiety: A review of self-reports

School anxiety is a set of cognitive, motor and psychophysiological responses emitted by a person in school situations that are perceived as a threat. The research objectives of this study were 


\section{ANSIEDAD ESCOLAR INFANTOJUVENIL: UNA REVISIÓN DE AUTOINFORMES}

two. The first one was to describe school anxiety self-reports that have been elaborated and/or validated between 2005 and 2015: School Anxiety Inventory, School Anxiety Inventory-Short Version, Children's Anxiety in Math Scale, and Test Anxiety Inventory for Children and Adolescents. The second aim was to analyze the reliability (internal consistency and temporal stability) and validity (factorial structure) of the inventories and the scale mentioned above. Results suggest that the psychometric properties of these instruments are suitable. Therefore, these can be used by clinical and educational professionals to assess the school anxiety of children and adolescents.

Key words: school anxiety, childhood, adolescence, assessment, self-report.

\section{ANTECEDENTES}

La ansiedad escolar fue definida por Kearney, Cook y Chapman (2007) como un conjunto de síntomas físicos y cognitivos de carácter desagradable que se manifiestan en respuesta a factores escolares estresantes, generales o concretos. Un año más tarde, García-Fernández, Inglés, MartínezMonteagudo y Redondo (2008) matizaron la concepción, introduciendo para este tipo de ansiedad los tres sistemas de respuesta de Lang (1968): cognitivo (pensamientos y aprensión desagradables), fisiológico (excitación elevada) y conductual (resistencia o huida), y señalando que las situaciones estresantes son percibidas como un riesgo por los sujetos afectados. Atendiendo a MartínezMonteagudo, Inglés, Trianes y García-Fernández (2011), estas circunstancias amenazadoras resultan ser con frecuencia las siguientes: el fracaso y castigo escolar, la agresión, la evaluación social en el contexto escolar, y la evaluación escolar.

Conviene apuntar que la ansiedad posee un carácter anticipatorio, en el sentido de que alerta al sujeto de posibles amenazas, por lo que puede considerarse funcional 0 adaptativa (GarcíaFernández, Martínez-Monteagudo e Inglés, 2013; Spielberger, 1979). Sin embargo, los síntomas ansiosos se manifiestan en distinto grado de persistencia e intensidad (Jadue, 2001), por lo que a niveles altos la ansiedad deja de ser necesaria y se convierte en desadaptativa, causando graves consecuencias en quienes la padecen. En este sentido, los niños y adolescentes con ansiedad escolar poseen disfunciones cognitivas en los procesos de atención y memoria, y profundas dificultades para concentrarse, por lo que su rendimiento académico se ve afectado (Jadue, 2001; Rains, 2004). Además, la presencia de la ansiedad escolar suele llevar asociada problemas como la depresión o la baja autoestima y derivar en trastornos afectivos y de ansiedad en la edad adulta (Bados, 2005). Por lo que respecta a la frecuencia de aparición, esta sintomatología desagradable y desadaptativa ante situaciones escolares consideradas como amenazantes, puede llegar a presentarse hasta en el $18 \%$ de los niños de entre 3 y 14 años (Bados, 2005; Granell de Aldaz, Vivas, Gelfand y Feldman, 1984; Orgilés, Espada, García-Fernández y Méndez, 2009; Méndez, 2005).

Teniendo en consideración el porcentaje de prevalencia y las repercusiones en la adultez señaladas anteriormente, se hace necesaria una evaluación temprana de la ansiedad escolar con instrumentos válidos y fiables, que permita ajustar el tratamiento al sujeto y aplicarlo lo antes posible. En cambio, examinando la literatura científica únicamente se ha encontrado un estudio (MartínezMonteagudo, Inglés y García-Fernández, 2013) que revise los autoinformes (cuestionarios, inventarios y escalas) que presentan unas propiedades psicométricas adecuadas para ser utilizados en los ámbitos clínico y educativo.

\section{OBJETIVOS}

El conocimiento científico acerca de cuáles son los instrumentos válidos y fiables que se pueden utilizar para evaluar la ansiedad escolar está limitado a la revisión de Martínez-Monteagudo et al. (2013), y esta alcanza aquellos cuestionarios, inventarios y escalas que fueron creadas y validadas hasta junio del año 2012. Por tanto, la presente investigación se propuso ampliar y actualizar 
el conocimiento sobre el tema partiendo de un doble objetivo: primeramente, describir los autoinformes elaborados y/o validados para la evaluación de la ansiedad escolar infantojuvenil entre los años 2005 y 2015; en segundo término, analizar la fiabilidad (consistencia interna y estabilidad temporal) y validez (estructura factorial) de estos cuestionarios, inventarios y escalas.

\section{MÉTODO}

\section{Procedimiento}

En primer lugar, se llevó a cabo la búsqueda de las unidades de análisis. Para ello, se determinaron las palabras clave y se diseñó la estrategia de búsqueda compuesta por los siguientes términos, truncadores y booleanos: anxiety $A N D$ (child* $O R$ adolescen ${ }^{*} O R$ teenage*) AND (index ${ }^{*} O R$ assessment $O R$ validation $O R$ "psychometric properties" $O R$ variable* $O R$ treat* $O R$ intervention). Acto seguido, se introdujo esta estrategia en las bases de datos Web of Science (todas las bases de datos), Scopus, PsycINFO y ERIC, acotando en todas ellas el periodo temporal entre el 2005 y el 2015. En el caso de PsycINFO y ERIC, se utilizó la opción «evaluado por expertos» con objeto de eliminar todos aquellos documentos que no hubiesen sido sometidos a una revisión por pares. Mediante esta estrategia, se obtuvieron un total de 1.567 resultados. Posteriormente, se aplicaron sucesivos criterios de exclusión para seleccionar la muestra (véase la Figura 1).

\section{Figura 1.}

Diagrama de flujo del proceso de selección de la muestra a través de diversas fases de exclusión de unidades

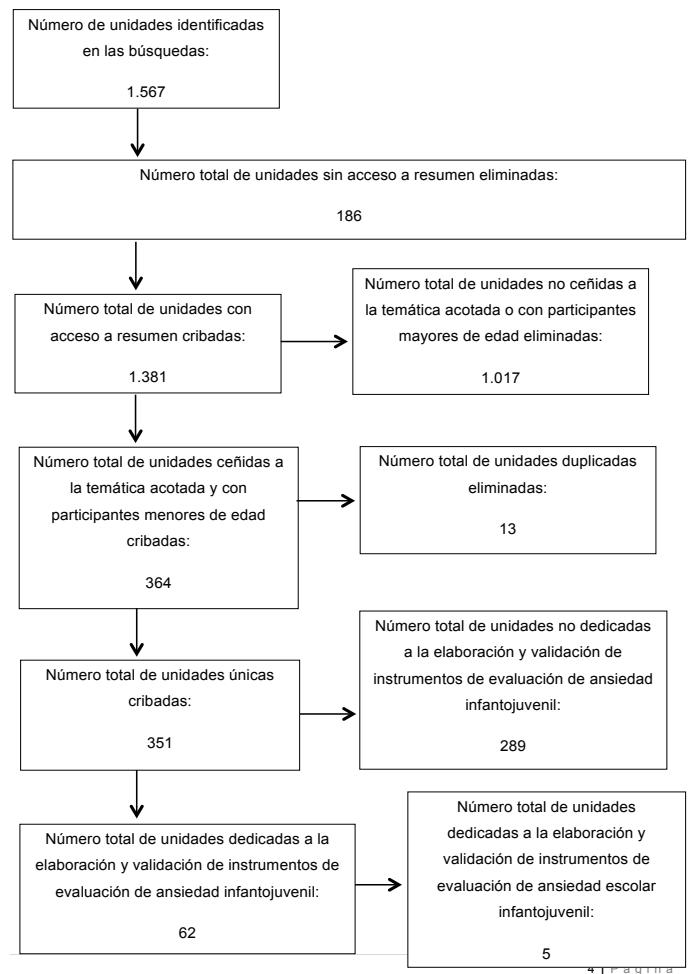




\section{ANSIEDAD ESCOLAR INFANTOJUVENIL: UNA REVISIÓN DE AUTOINFORMES}

Tras el proceso de selección, se obtuvieron 5 trabajos. Conviene destacar que dos de ellos tenían por objeto validar una misma escala en poblaciones diferentes, por lo que se rastrearon las referencias de los artículos correspondientes y se localizó el documento del instrumento original, no devuelto por las bases de datos en la búsqueda inicial. Así se obtuvo un conocimiento completo de los avances en el perfeccionamiento de esta escala, tales como el cambio en el número de ítems.

\section{Materiales}

El número de trabajos correspondientes a la creación de instrumentos originales fue 1. Sumando este a los 5 seleccionados previamente, se obtuvo una muestra conformada finalmente por 6 elementos (que presentaban un total de 4 autoinformes). Cabe destacar que, si bien no se limitó el tipo de documento, las 6 unidades de análisis fueron artículos de investigación.

\section{RESULTADOS}

Una única herramienta ha sido desarrollada para evaluar la ansiedad que experimenta un sujeto cuando se expone a diferentes situaciones escolares. Este es el Inventario de Ansiedad Escolar (IAES) de García-Fernández, Inglés, Martínez-Monteagudo, Marzo y Estévez (2011), el cual fue simplificado en el School Anxiety Inventory-Short Version (SAI-SV) por García-Fernández, Inglés, Marzo y Martínez-Monteagudo (2014), para poder ser implementado en situaciones en las que se dispone de un período corto de tiempo.

Por otra parte, conviene añadir la Children's Anxiety in Math Scale (Jameson, 2013), pues si bien no contempla circunstancias escolares en general, sí evalúa las que tienen lugar en relación con el área de Matemáticas. Asimismo, únicamente ha sido encontrado un instrumento que evalúe la ansiedad que experimentan infantes y adolescentes ante situaciones de examinación: el Test Anxiety Inventory for Children and Adolescents (TAICA) de Lowe y Lee (2004). Cabe destacar que ha sido validado dos veces: la primera, por Lowe et al. (2008) y la segunda, por Unruh y Lowe (2010); en esta última se llevó a cabo una reducción del número de ítems.

En la Tabla 1 se recogen los instrumentos mencionados, especificando para cada uno de ellos el nombre, el autor 0 autores y el año de publicación, el rango de edad de sus destinatarios, el número de ítems por el que está formado, los factores o variables que lo componen, y la escala de respuesta. Asimismo, incluye los datos referentes al análisis de la validez: el tipo de análisis y rotación factorial utilizados, y al análisis de la fiabilidad: la consistencia interna (alpha de Cronbach) y la estabilidad temporal (test-retest).

Tabla 1

Inventarios y escalas de ansiedad escolar

\begin{tabular}{|c|c|c|c|c|c|c|c|c|c|}
\hline Instrumento & Autor(es) & Edad & $\begin{array}{l}N^{\circ} \text { de } \\
\text { items }\end{array}$ & $\begin{array}{l}\text { Factores o } \\
\text { variables }\end{array}$ & Escala & $\begin{array}{l}\text { Análisis } \\
\text { factorial }\end{array}$ & Rotación & $\begin{array}{l}\text { Alpha total } \\
\text { (escalas) }\end{array}$ & Test-retest \\
\hline $\begin{array}{l}\text { Inventario } \\
\text { de Ansiedad } \\
\text { Escolar } \\
\text { (IAES) }\end{array}$ & $\begin{array}{l}\text { García- } \\
\text { Fernández, } \\
\text { Inglés, } \\
\text { Martínez- } \\
\text { Monteagudo, } \\
\text { Marzo y } \\
\text { Estévez } \\
\text { (2011) }\end{array}$ & $\begin{array}{l}12- \\
18 \\
\text { años }\end{array}$ & 55 & $\begin{array}{l}\text { Situaciones } \\
\text { escolares: } \\
\text {--Ansiedad ante } \\
\text { el } \\
\text { fracaso y } \\
\text { castigo escolar } \\
\text {--Ansiedad ante } \\
\text { la agresión } \\
\text { - Ansiedad } \\
\text { ante la } \\
\text { evaluación } \\
\text { social } \\
\text {.-Ansiedad } \\
\text { ante la } \\
\text { evaluación } \\
\text { escolar } \\
\text { Sistemas de } \\
\text { respuesta de } \\
\text { ansiedad: } \\
\text {--Ansiedad } \\
\text { cognitiva } \\
\text {--Ansiedad } \\
\text { psicofisiológica } \\
\text {--Ansiedad } \\
\text { conductual }\end{array}$ & $\begin{array}{l}0= \\
\text { Nunca a } \\
4= \\
\text { Siempre }\end{array}$ & $\begin{array}{l}\text { AFE Y } \\
\text { AFC }\end{array}$ & Varimax & $\begin{array}{l}.93 \\
\text { (Ansiedad } \\
\text { ante la } \\
\text { evaluación } \\
\text { social),. } 92 \\
\text { (Ansiedad } \\
\text { ante el } \\
\text { fracaso } \\
\text { escolar y el } \\
\text { castigo } \\
\text { escolar y } \\
\text { Ansiedad } \\
\text { ante la } \\
\text { agresión), } \\
.88 \\
\text { (Ansiedad } \\
\text { ante la } \\
\text { evaluación } \\
\text { escolar), } \\
.86 \\
\text { (Ansiedad } \\
\text { cognitiva y } \\
\text { Ansiedad } \\
\text { psicofi } \\
\text { siológica) }\end{array}$ & $\begin{array}{l}.84 \\
\text { (Ansiedad } \\
\text { ante el } \\
\text { fracaso y } \\
\text { castigo } \\
\text { escolar y } \\
\text { Ansiedad } \\
\text { ante } \\
\text { la } \\
\text { evaluación } \\
\text { social), .83 } \\
\text { (Ansiedad } \\
\text { ante la } \\
\text { evaluación } \\
\text { escolar), } \\
.78 \\
\text { (Ansiedad } \\
\text { ante la } \\
\text { agresión), } \\
.77 \\
\text { (Ansiedad } \\
\text { cognitiva), } \\
.75 \\
\text { (Ansiedad } \\
\text { psicofi }\end{array}$ \\
\hline
\end{tabular}




\begin{tabular}{|c|c|c|c|c|c|c|c|c|c|}
\hline & & & & & & & & $\begin{array}{l}\text { y. } 82 \\
\text { (Ansiedad } \\
\text { conductual) }\end{array}$ & $\begin{array}{l}\text { siológica) y } \\
.74 \\
\text { (Ansiedad } \\
\text { conductual) } \\
\text { (2 } \\
\text { semanas) }\end{array}$ \\
\hline $\begin{array}{l}\text { School } \\
\text { Anxiety } \\
\text { Inventory- } \\
\text { Short } \\
\text { Version } \\
\text { (SAI-SV) }\end{array}$ & $\begin{array}{l}\text { García- } \\
\text { Fernández, } \\
\text { Inglés, Marzo } \\
\text { y Martínez- } \\
\text { Monteagudo } \\
\text { (2014) }\end{array}$ & $\begin{array}{l}12- \\
18 \\
\text { años }\end{array}$ & 116 & $\begin{array}{l}\text { Situaciones } \\
\text { escolares: } \\
\text {-Ansiedad ante } \\
\text { la agresión } \\
\text {-Ansiedad ante } \\
\text { la evaluación } \\
\text { social } \\
\text {--Ansiedad ante } \\
\text { el fracaso } \\
\text { académico } \\
\text { Sistemas de } \\
\text { respuesta de } \\
\text { ansiedad: } \\
\text {-Ansiedad } \\
\text { fisiológica } \\
\text {-Ansiedad } \\
\text { cognitiva } \\
\text {-Ansiedad } \\
\text { conductual }\end{array}$ & $\begin{array}{l}0= \\
\text { Nunca a } \\
4= \\
\text { Siempre }\end{array}$ & $\begin{array}{l}\text { AFE y } \\
\text { AFC }\end{array}$ & Promax & $\begin{array}{l}.94 \text { (AA), } \\
.91 \\
\text { (AES), } .89 \\
\text { (AFA), .84 } \\
\text { (AF), } 85 \\
\text { (AC), y.77 } \\
\text { (AC) }\end{array}$ & $\begin{array}{l}.87(\mathrm{AA}), \\
.85 \\
(\mathrm{AES}), .83 \\
(\mathrm{AFA}), .78 \\
(\mathrm{AF}), .76 \\
(\mathrm{AC}), \text { and } \\
.74(\mathrm{AC}) \\
\text { (2 } \\
\text { semanas) }\end{array}$ \\
\hline $\begin{array}{l}\text { Children's } \\
\text { Anxiety in } \\
\text { Math Scale }\end{array}$ & $\begin{array}{l}\text { Jameson } \\
(2013)\end{array}$ & $\begin{array}{l}\text { 6-10 } \\
\text { años }\end{array}$ & 16 & $\begin{array}{l}\text { Realización de } \\
\text { las Matemáticas } \\
\text { Realización de } \\
\text { las Matemáticas } \\
\text { en Situaciones } \\
\text { Sociales } \\
\text { Pensamiento } \\
\text { Matemático } \\
\text { Esfuerzo en } \\
\text { Matemáticas }\end{array}$ & $\begin{array}{l}\text { 5= Muy } \\
\text { ansioso } \\
\text { a } 1=N o \\
\text { ansioso } \\
\text { en } \\
\text { absoluto }\end{array}$ & AFE & Varimax & .86 & --- \\
\hline $\begin{array}{l}\text { Test Anxiety } \\
\text { Inventory for } \\
\text { Children and } \\
\text { Adolescents } \\
\text { (TAICA) }\end{array}$ & $\begin{array}{l}\text { Lowe y Lee } \\
(2004)\end{array}$ & $\begin{array}{l}9-19 \\
\text { años }\end{array}$ & 45 & $\begin{array}{l}\text { Obstrucción } \\
\text { cognitiva/ } \\
\text { Inatención } \\
\text { Hiperactivación } \\
\text { Fisiológica } \\
\text { Humillación } \\
\text { social } \\
\text { Preocupación } \\
\text { Mejora de } \\
\text { Rendimiento/ } \\
\text { Facilitación de } \\
\text { Ansiedad } \\
\text { Mentira }\end{array}$ & $\begin{array}{l}1= \\
\text { Nunca } \\
\text { es cierto } \\
\text { acerca } \\
\text { de mí, a } \\
5= \\
\text { Siempre } \\
\text { es cierto } \\
\text { acerca } \\
\text { de mí }\end{array}$ & $\begin{array}{l}\text { AFE y } \\
\text { ACP }\end{array}$ & Promax & $(.77-.90)$ & -- \\
\hline Validación & $\begin{array}{l}\text { Lowe, } \\
\text { Lee, } \\
\text { Witteborg, } \\
\text { Prichard, } \\
\text { Luhr, } \\
\text { Cullinan, } \\
\text { Mildren, } \\
\text { Raad, } \\
\text { Cornelius y } \\
\text { Janik (2008) }\end{array}$ & $\begin{array}{l}9-19 \\
\text { años }\end{array}$ & 45 & $\begin{array}{l}\text { Obstrucción } \\
\text { cognitiva/ } \\
\text { Inatención } \\
\text { Hiperactivación } \\
\text { Fisiológica } \\
\text { Humillación } \\
\text { social } \\
\text { Preocupación } \\
\text { Mejora de } \\
\text { Rendimiento/ } \\
\text { Facilitación de } \\
\text { Ansiedad } \\
\text { Mentira }\end{array}$ & $\begin{array}{l}1= \\
\text { Nunca } \\
\text { es cierto } \\
\text { acerca } \\
\text { de mí, a } \\
5= \\
\text { Siempre } \\
\text { es cierto } \\
\text { acerca } \\
\text { de mí }\end{array}$ & --- & --- & $(.81-.94)$ & $\begin{array}{l}(.81-90) \\
\text { (7-21 dias) }\end{array}$ \\
\hline $\begin{array}{l}\text { Validación } \\
\text { española }\end{array}$ & $\begin{array}{l}\text { Unruh y Lowe } \\
(2010)\end{array}$ & $\begin{array}{l}\text { 9-19 } \\
\text { años }\end{array}$ & 31 & $\begin{array}{l}\text { Obstrucción } \\
\text { cognitiva/ } \\
\text { Inatención } \\
\text { Hiperactivación } \\
\text { Fisiológica } \\
\text { Humillación } \\
\text { social } \\
\text { Preocupación } \\
\text { Mejora de } \\
\text { Rendimiento/ } \\
\text { Facilitación de } \\
\text { Ansiedad } \\
\text { Mentira }\end{array}$ & $\begin{array}{l}1= \\
\text { Nunca } \\
\text { es cierto } \\
\text { acerca } \\
\text { de mí, a } \\
5= \\
\text { Siempre } \\
\text { es cierto } \\
\text { acerca } \\
\text { de mí }\end{array}$ & AFE & Promax & $(.68-.93)$ & -- \\
\hline
\end{tabular}

\section{CONCLUSIONES}

El objetivo del presente estudio fue revisar los autoinformes de ansiedad escolar que han sido creados y/o validados dentro del periodo de tiempo comprendido entre el año 2005 y el 2015, y analizar la fiabilidad y validez de las mismas. Del proceso investigador llevado a cabo se ha obtenido que en la actualidad únicamente se dispone de un instrumento que evalúa la ansiedad escolar en niños y adolescentes: el Inventario de Ansiedad Escolar (IAES) de García-Fernández et al. (2011), con su correspondiente versión corta llamada: School Anxiety Inventory-Short Version (SAI-SV), de García-Fernández et al. (2014). Asimismo, se han identificado otros autoinformes que evalúan los niveles de ansiedad de los sujetos ante aspectos concretos del ámbito escolar: la Children's Anxiety in Math Scale (Jameson, 2013) para el área de Matemáticas, y el Test Anxiety Inventory for Children 


\section{ANSIEDAD ESCOLAR INFANTOJUVENIL: UNA REVISIÓN DE AUTOINFORMES}

and Adolescents (TAICA) de Lowe y Lee (2004) para la ansiedad ante los exámenes. Conviene señalar que el IAES (García-Fernández et al., 2011) es el único autoinforme coincidente con los resultados de la revisión, sobre instrumentos de ansiedad escolar, que llevaron a cabo previamente a este estudio Martínez-Monteagudo et al. (2013).

Por lo que respecta a la evaluación específica de cada uno de los tres sistemas de respuesta de la ansiedad: cognitivo, fisiológico y conductual, de los 4 instrumentos únicamente el IAES (MartínezMonteagudo et al., 2013) y el SAI-SV (García-Fernández et al., 2014) la llevan a cabo. Se considera oportuno añadir al respecto que ninguno de los tres sistemas resulta prioritario en relación con la respuesta compleja de miedo 0 ansiedad; cada uno de ellos es independiente y posee una gran relevancia a la hora de valorar los síntomas (Lang, 1968). Por el contrario, la Children's Anxiety in Math Scale (Jameson, 2013) y el TAICA (Lowe y Lee, 2004) no proporcionan información al respecto, ofreciendo así una única puntuación.

Otro aspecto a considerar es que los índices de consistencia interna y de fiabilidad test-retest resultan adecuados para los autoinformes correspondientes, atendiendo a los criterios determinados por Prieto y Muñiz (2000). No obstante, la fiabilidad test-retest se ha evaluado en intervalos de tiempo demasiado cortos, siendo el más amplio el correspondiente a la validación del TAICA realizada por Lowe et al. (2008) que comprende de 7 a 21 días. Por tanto, pese a que los índices obtenidos se pueden considerar aceptables, para futuras investigaciones se recomienda utilizar intervalos de meses; esto permitirá comprobar si los instrumentos son realmente estables temporalmente.

Los resultados obtenidos permiten concluir que los autoinformes presentan propiedades psicométricas adecuadas para poder ser utilizados por profesionales de la psicología clínica y educativa, en el proceso de valoración de la ansiedad escolar de infantes y adolescentes. No obstante, se han identificado algunas deficiencias en relación con las variables que evalúan y el proceso de análisis de fiabilidad llevado a cado, que convendría tener en cuenta de cara a la elaboración de nuevos instrumentos de evaluación de la ansiedad escolar 0 a las próximas validaciones de los ya existentes.

Por último, se considera oportuno destacar las limitaciones del presente estudio que han sido detectadas. En primer lugar, la acotación del periodo temporal al efectuar la búsqueda en las diferentes bases de datos utilizadas, ha podido excluir trabajos que se ajustan a la estrategia diseñada pero que han sido publicados posteriormente. En segundo lugar, la selección de unos determinados términos en el diseño de la estrategia de búsqueda puede haber descartado otros estudios pertinentes y relevantes en esta revisión autoinformes. Pese a todo lo anterior, el presente estudio ha proporcionado una actualización de los instrumentos que son válidos y fiables para evaluar la ansiedad infantojuvenil, haciéndose así más amplio y consistente el conocimiento científico al respecto.

\section{REFERENCIAS}

Bados, A. (2005). Trastorno de ansiedad por separación. Rechazo escolar y fobia escolar. Facultad de Psicología, Universidad de Barcelona.

García-Fernández, J. M., Inglés, C. J., Martínez-Monteagudo, M. C., Marzo, J. y Estévez, E. (2011). Inventario de Ansiedad Escolar: validación en una muestra de estudiantes de Educación Secundaria. Psicothema, 23(2), 301-307.

García-Fernández, J. M., Inglés, C. J., Martínez-Monteagudo, M. C. y Redondo, J. (2008). Evaluación y tratamiento de la ansiedad escolar en la infancia y la adolescencia. Psicología Conductual, 16, 413-437.

García-Fernández, J. M., Inglés, C. I., Marzo, J. C. y Martínez-Monteagudo, M. C. (2014). Psychometric properties of the School Anxiety Inventory-Short Version in Spanish secondary 
education students. Psicothema, 26(2), 286-292. doi: 10.7334/psicothema2013.288

García-Fernández, J. M., Martínez-Monteagudo, M. C., y Inglés, C. J. (2013). ¿Cómo se relaciona la ansiedad escolar con el rendimiento académico? Revista Iberoamericana de Psicología y Salud, 4(1), 63-76.

Granell de Aldaz, E., Vivas, E., Gelfand, D. y Feldman, L. (1984). Estimating the prevalence of school refusal and school-related fears. A Venezuelan sample. Journal of Nervous and Mental Disease, 172, 722-729.

Jadue, G. (2001). Algunos efectos de la ansiedad en el rendimiento escolar. Estudios pedagógicos, 27, 111-118. doi: 10.4067/S0718-07052001000100008

Jameson, M. M. (2013). The Development and Validation of the Children's Anxiety in Math Scale. Journal of Psychoeducational Assessment, 31(4), 391-395. doi: 10.1177/0734282912470131

Kearney, C. A., Cook, L. C. y Chapman, G. (2007). School stress and school refusal behavior. En G. Fink (Ed.), Encyclopedia of stress (2a ed.) (Vol. 3) (pp. 422-425). San Diego, CA: Academic Press.

Lang, P. J. (1968). Fear reduction and fear behavior: Problems in treating a construct. En J. U. H. Shilen (Ed.), Research in psychotherapy, vol. 3 (pp. 90-102). Washington: American Psychological Association.

Lowe, P. A. y Lee, S. W. (2004). Test Anxiety Inventory for Children and Adolescents. Lawrence, KS: University of Kansas.

Lowe, P. A., Lee, S. W., Witteborg, K. M., Prichard, K. W., Luhr, M. E., Cullinan, ... Janik, M. (2008). The Test Anxiety Inventory for Children and Adolescents (TAICA): Examination of the Psychometric Properties of a New Multidimensional Measure of Test Anxiety Among Elementary and Secondary School Students. Journal of Psychoeducational Assessment, 26(3), 215-230. doi: 10.1177/0734282907303760

Martínez-Monteagudo, M. C. Inglés, C. Y García-Fernández, J. M. (2013). Evaluación de la ansiedad escolar: revisión de cuestionarios, inventarios y escalas. Psicología Educativa, 19, 27-36. doi: 10.5093/ed2013a5

Martínez-Monteagudo, M. C., Inglés, C. J., Trianes, M. V. y García-Fernández, J. M. (2011). Perfiles de ansiedad escolar: Diferencias en Clima Social y Violencia entre Iguales. Electronic Journal of Research in Educational Psychology, 9(3), 1023-1042.

Méndez, F. X. (2005). Miedos y temores en la infancia: Ayudar a los niños a superarlos. Madrid: Pirámide.

Orgilés, M., Espada, J. P., García-Fernández, J. M. y Méndez, F. X. (2009). Relación entre miedos escolares y síntomas de ansiedad por separación. Revista Mexicana de Psicología, 26, 17-25.

Prieto, G. y Muñiz, J. (2000). Un modelo para evaluar la calidad de los tests utilizados en España. Papeles del Psicólogo, 77, 65-75.

Rains, D. (2004). Principios de neuropsicología humana. México: McGraw-Hill.

Spielberger, C. (1979). Tensión y ansiedad. México: Harla S. A.

Unruh, S. M. y Lowe, P. A. (2010). The Development and Validation of a Spanish Language Version of the Test Anxiety Inventory for Children and Adolescents. Hispanic Journal of Behavioral Sciences, 32(1), 164-183. doi: 10.1177/0739986309355129 
